\title{
Mechanical Analysis of Encapsulated Metal Interconnects under Transversal Load
}

\author{
B. Van Keymeulen ${ }^{1}$, M. Gonzalez ${ }^{2}$, F. Bossuyt ${ }^{1}$, J. De Baets $^{1}$, and J. Vanfleteren ${ }^{1}$ \\ ${ }^{1}$ Centre for Microsystems Technology (CMST), imec and Ghent University, Technologiepark 914a, 9052 Gent, \\ Belgium \\ 2 imec, Kapeldreef 75, 3001 Leuven, Belgium
}

\begin{abstract}
Novel insights regarding the ability of encapsulated metal interconnections to deform due to bending are presented. Encapsulated metal interconnections are used as electric conductor or measurement system within a wide range of applications fields, e.g. biomedical, wearable, textile applications. Nevertheless the mechanical analysis remains limited to reliability investigation of these configurations while deformability is as important for application fields where, so-called disappearing electronics is the main purpose.
\end{abstract}

An analysis based on the work needed to bend interconnections to a certain curvature will be used to compare different interconnection configurations with each other. The experimental as well as the simulation setup is based on PDMS encapsulated PI-enhanced $\mathrm{Cu}$ tracks. The results and conclusions are specific for this type of interconnections, but can be extended to a global conclusion about stretchable interconnections.

From the obtained insights it is proven that periodically meander-shaped interconnections need significant less work, up to more than 10 times less, to bend the interconnection to the same curvature compared to straight interconnection lines. Furthermore, the bendability of the straight interconnection lines is determined by the shape of the interconnection, where for meandered tracks the encapsulation will determine this factor, for an encapsulation thickness of maximum $1 \mathrm{~mm}$. It shows out, for the meander-shaped interconnection, that per increase of $250 \mu \mathrm{m}$ encapsulation thickness the work raises with a factor 2 . For straight interconnection lines the work in function of the encapsulation thickness is limited to $20 \% / 250 \mu \mathrm{m}$. For encapsulations $>1 \mathrm{~mm}$, the encapsulation thickness will become the predominant factor which determines the deformability for both interconnection shapes.

\section{Introduction}

Encapsulated metal interconnections are widely investigated and used within applications where a certain degree of comfort is necessary from the user side of view or where deformability, one-time or more, is a necessary feature [1].

The traditional way to obtain electronic functionalities is to making use of conventional rigid PCBs. These kind of electronic circuits are not deformable from nature and don't accomplish the previous mentioned features regarding comfort and deformability.

Less traditional is the use of flexible circuit boards. These boards are bendable, which brings along a certain degree of comfort and deformability when one applies them in a bendable application, e.g. shoe sole.

Last years a novel approach has been invented, investigated and implemented, namely the stretchable circuit board technology. Here the interconnections are not only bendable, they are also stretchable and can be torsed without causing permanent damage under certain, limited, mechanical load conditions.

The deformability of the stretchable interconnections is widely investigated and reported in numerous papers by different research groups and institutes [2-8]. This gives us insights in design rules for reliable interconnections under, what will be called in this paper, longitudinal load conditions. Where one has now insights in developing reliable structures, one doesn't know how much work is needed to deform these kinds of interconnections. For this reason the stretchable encapsulated interconnection, made with meandered PI-supported copper tracks, will be compared with a flexible encapsulated interconnection, built up from the same material to obtain an objective comparison between both structures. Flexible interconnections are investigated because of the very simple design rules in comparison with meandered tracks and to obtain knowledge about the mechanical features of both configurations, which was not reported till now. Remark that 2D deformable structures can be very useful in certain devices, e.g. textile applications where the textile is typical not stretchable. If stretchable interconnections don't deliver additional advantages in such a case, flexible tracks can be used for their simplicity. It will be shown that unless the fact those stretchable as well as flexible interconnections are both 2D deformable, stretchable electronics delivers significant advantages regarding the ability to deform under a transversal load.

Because of the fact that flexible interconnections can't be stretched due to their straight nature, one other load case is needed instead of elongation to compare both kinds of structures. For this reason, and due to the fact that stretchable as well as flexible interconnections are bendable, a test environment will be used where the interconnections are bent to a certain curvature, while monitoring the delivered work to obtain this curvature.

The results of the numerical simulation are validated by practical experiments. 
Stretchable interconnections are not only having the ability to deform in longitudinal direction where flexible electronics don't have this ability. But, and this is the major observation of this investigation, stretchable electronics are more deformable, if they are bended, in comparison with flexible interconnections. From this it can be concluded that stretchable interconnections have advantages over flexible interconnections to implement in deformable but not necessarily extensible structures where comfort is a necessity, e.g. textile applications.

Furthermore, previous investigations [10] report that the thicker the encapsulation is, the more reliable a certain structure will behave. In this paper it is proven and validated that increasing the encapsulation thickness brings along a significant decrease in deformability. The flexural rigidity increases with a factor two per $250 \mu \mathrm{m}$ additional encapsulation thickness in case of meander shaped interconnections. This brings along a trade-off between reliability and deformability/comfort.

Unless the fact that the obtained results corresponds with PI-supported copper tracks, the conclusions can be transferred to other products serving as conductive material, encapsulation material and support material.

\section{Technology description}

This section summarizes the production method for the practical test vehicles used within this investigation. The production method for stretchable circuitry described in [7] will be used as starting point. Nevertheless some processing steps can be left out because only interconnections and not a whole circuitry is needed.

The used stack is a copper track supported with a layer of PI. The layer of PI has a certain offset in comparison with the copper track. The PI support layer is needed to enhance the life time of the metal interconnection under longitudinal load conditions as elongation, [9].

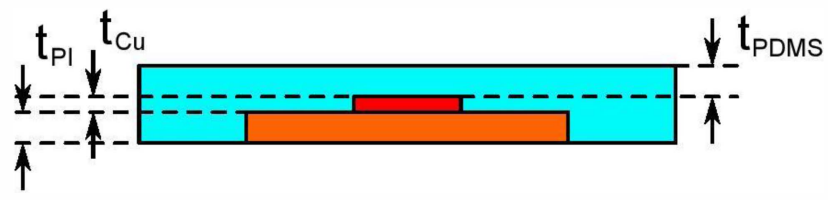

\section{Figure 1: wanted stack of material within encapsulated PI-supported copper interconnection lines}

For completeness the corresponding thicknesses for the different materials are summarized in Table 1.

\begin{tabular}{ll}
\hline Material & Thickness \\
\hline $\mathrm{PDMS}$ & $0 \mu \mathrm{m}-2000 \mu \mathrm{m}$ \\
\hline $\mathrm{Cu}$ & $18 \mu \mathrm{m}$ \\
\hline $\mathrm{PI}$ & $35 \mu \mathrm{m}$ \\
\hline Table 1: material parameters in the height direction
\end{tabular}

Table 1: material parameters in the height direction
The processing starts with a flexible foil built up of PI with $\mathrm{Cu}$ on top of it. The copper has a thickness of $18 \mu \mathrm{m}$ where the PI has a thickness of $35 \mu \mathrm{m}$. This flexible foil needs to be processed following a conventional PCB manufacturing flow. The flexible foil is added to a rigid carrier to survive all the processing steps and to obtain an accurate product in the end. For this reason the flexible foil will be added to an FR4 board by means of a wax layer and vacuum lamination.

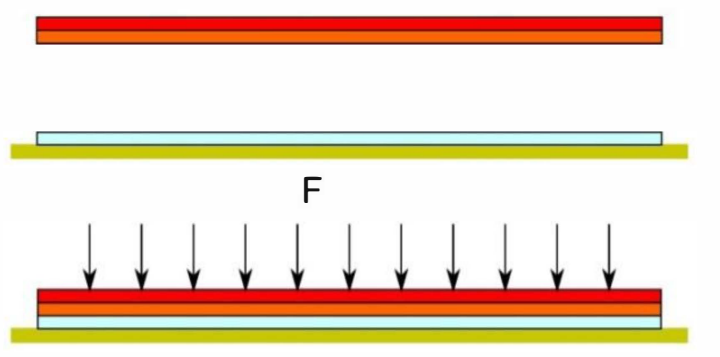

Figure 2: fix flexible foil to rigid carrier by wax layer

After adding the flexible foil on the FR4-board the structure undergoes a photolithography and etching process. We end up with a foil of PI with structured $\mathrm{Cu}$ at the locations where we want to have the interconnection lines.

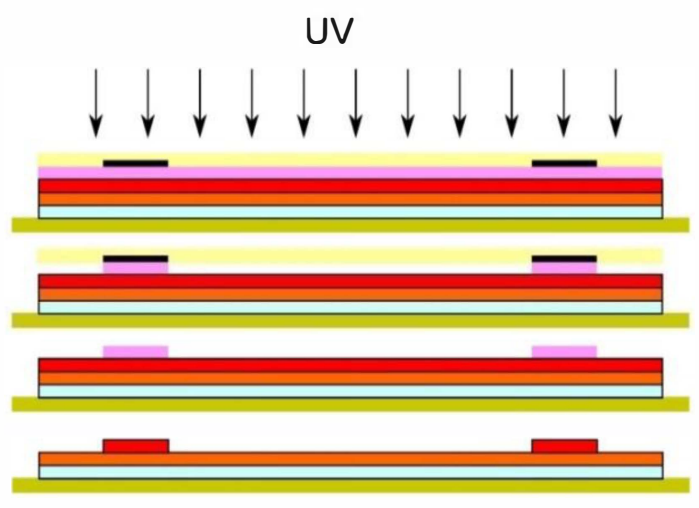

Figure 3: photolithography and etching

The PI layer will now be structured by means of a YAG-laser process. After cutting the PI, the residual PI is removed from the rigid board by means of manual peeling.

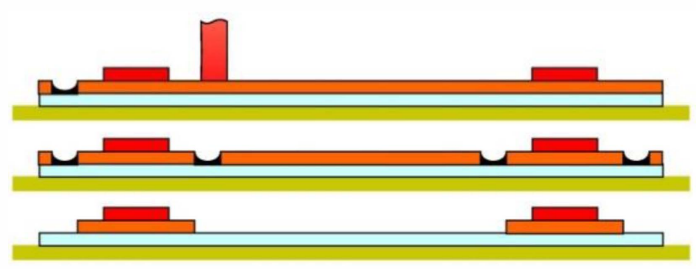


The global structure will be covered with a layer of PDMS, Sylgard 186, by means of doctor blading. The whole specimen is cured for 6 hours on a temperature of $50^{\circ} \mathrm{C}$. From now on the encapsulation is cured and the configurations can be cut out by hand or by laser ablation.

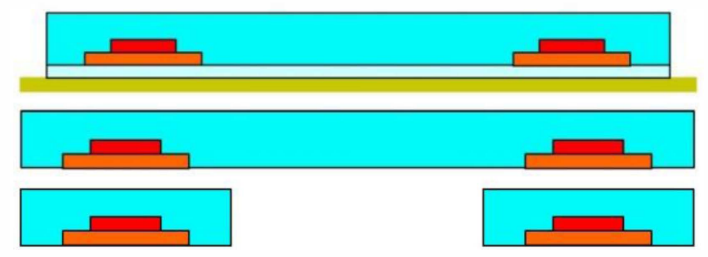

Figure 5: casting encapsulation and cutting structure

The resulting structures can be seen in Figure 6 for the flexible as well as for the meandered metal interconnections.

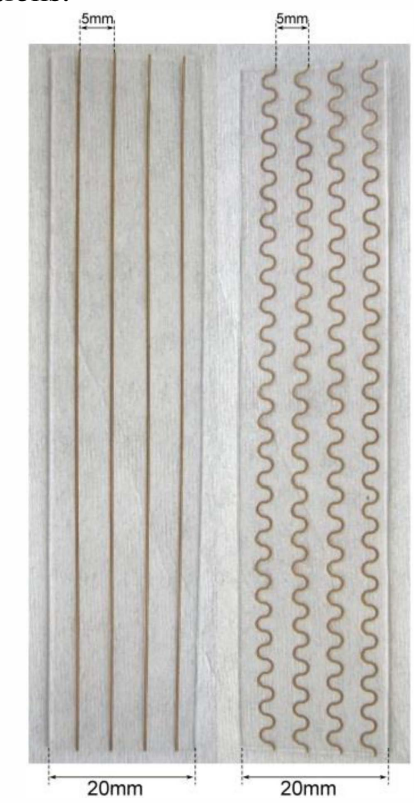

Figure 6: resulting samples (a) straight tracks (b) meandered tracks

\section{Test vehicle description}

\section{A. Flexible interconnection}

The outlines of a flexible interconnection are depicted in Figure 7 as model for numerical analysis (a) and as practical test vehicle (b). It exists of a straight track of $\mathrm{Cu}$ with a width of $100 \mu \mathrm{m}$. The PI has an offset of $100 \mu \mathrm{m}$ at both sides of the $\mathrm{Cu}$ track, resulting in a PI width of $300 \mu \mathrm{m}$. Furthermore the test vehicle will exist of 4 parallel tracks with a distance of $5 \mathrm{~mm}$ between the middle of the interconnect lines, as can be seen in Figure 6(a).

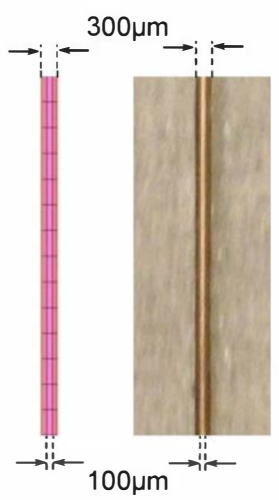

Figure 7: test vehicle for straight tracks (a) model (b) practical

\section{B. Stretchable interconnection}

The width of the copper as well as the offset of the PI support layer remains the same as for the straight tracks. The distance between the center of two meandered tracks remains also $5 \mathrm{~mm}$. The meander exists of two adjacent circles with an angle of $0^{\circ}$ between the centers of two adjacent circles. Furthermore the R/W ratio of the copper tracks is 13 . This makes the $\mathrm{R}=1,3 \mathrm{~mm}$.

For completeness a picture of the produced sample as well as the simulated design are depicted in Figure 8 .

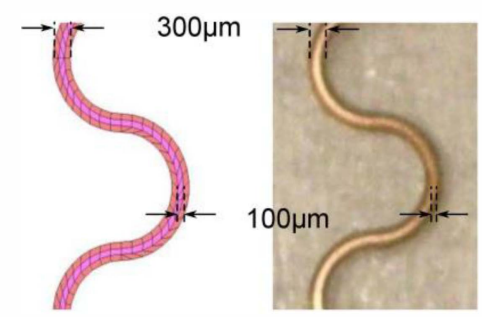

Figure 8: test vehicle for meandered tracks (a) model (b) practical

\section{Summary}

The dimensions of the model for numerical analysis are summarized in Table 2. The values are also indicated in Figure 6.

\begin{tabular}{lll}
\hline Parameter & $\begin{array}{l}\text { Flexible } \\
\text { interconnection }\end{array}$ & $\begin{array}{l}\text { Stretchable } \\
\text { interconnection }\end{array}$ \\
\hline Length sample & $108 \mathrm{~mm}$ & $108 \mathrm{~mm}$ \\
\hline Width sample & $20 \mathrm{~mm}$ & $20 \mathrm{~mm}$ \\
\hline Width $\mathrm{Cu}$ track & $0,1 \mathrm{~mm}$ & $0,1 \mathrm{~mm}$ \\
\hline Width PI track & $0,3 \mathrm{~mm}$ & $0,3 \mathrm{~mm}$ \\
\hline Spacing tracks & $5 \mathrm{~mm}$ & $5 \mathrm{~mm}$ \\
\hline Inner radius $\mathrm{Cu}$ & $1,3 \mathrm{~mm}$ & $\mathrm{NA}$ \\
\hline Table 2: design parameters test vehicles
\end{tabular}




\section{3D numerical simulation}

\section{A. Setup}

A schematic illustration of the simulation setup is shown in Figure 9. The right as well as the left downside of the encapsulated interconnection has a limited degree of freedom and can only move in the $\mathrm{x}$ direction.

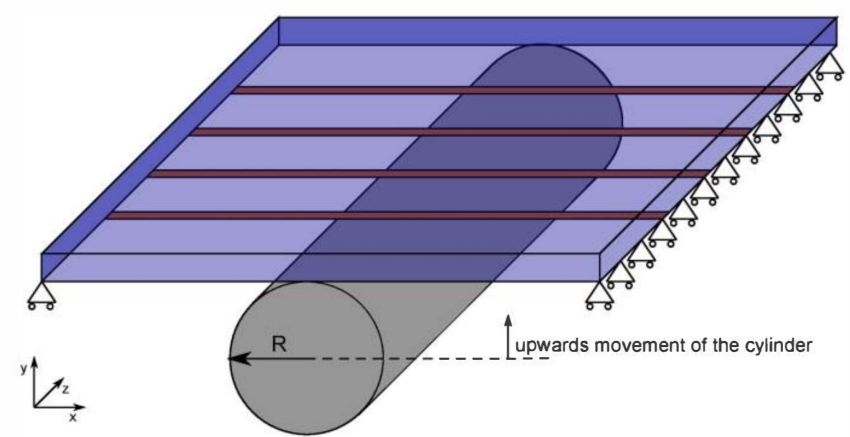

Figure 9: schematic overview simulation setup

A cylinder with a radius of $5 \mathrm{~mm}$ will move upwards with a constant speed, 2,5mm/iteration, and will be the load for the interconnection. A total of 10 iterations, or an upwards movement of $25 \mathrm{~mm}$ of the cylinder, is investigated.

The radius of the cylinder, Figure 9 and Figure $10(a)$, is rather small in comparison with the bending radius of the interconnection, see Figure 10(b) and Figure 10(c). The reason behind this choice in simulation environment is due to the fact that we want to investigate the ability of the interconnection to bend. If a cylinder is chosen with a bigger radius, friction effects will have an influence on this test environment and the structure will be stretched where we want to neglect this property in this investigation. Furthermore, moving the cylinder too much in the upwards direction, relative to the length of the interconnection, would also bring friction and elongation effects along.

The used material parameters are based on [10] and are summarized in Table 3.

\begin{tabular}{llll}
\hline Material & $\begin{array}{l}\text { Young's } \\
\text { modulus }\end{array}$ & $\begin{array}{l}\text { Poisson } \\
\text { ratio }\end{array}$ & $\mathrm{C}_{10}$ \\
\hline $\mathrm{PDMS}$ & $\mathrm{NA}$ & 0.5 & 0,156 \\
\hline $\mathrm{Cu}$ & $117 \mathrm{GPa}$ & 0,35 & $\mathrm{NA}$ \\
\hline $\mathrm{PI}$ & $3,2 \mathrm{GPa}$ & 0,34 & $\mathrm{NA}$ \\
\hline
\end{tabular}

Table 3: modelling parameters

The cylinder used to apply the load only touches the middle of the interconnection, which brings along a certain reaction force at the left and right side of the interconnection. This typical structure will now act as what is called in literature [11], a nonlinear stiffening spring.

Figure 10 shows the simulated behavior of the interconnection under bending. It can be seen that the higher the cylinder pushes the structure, the smaller its bending radius, or the higher the curvature, which is defined as $\kappa=\frac{1}{R}$.
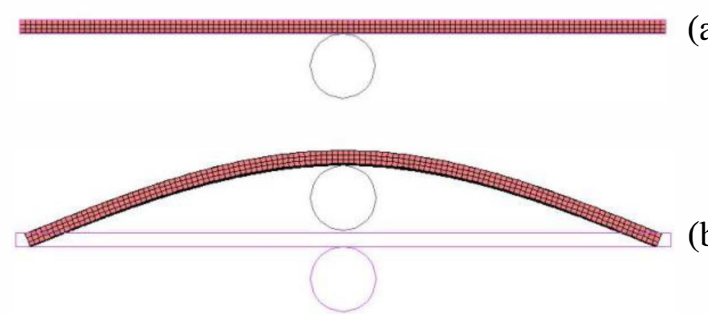

(b)

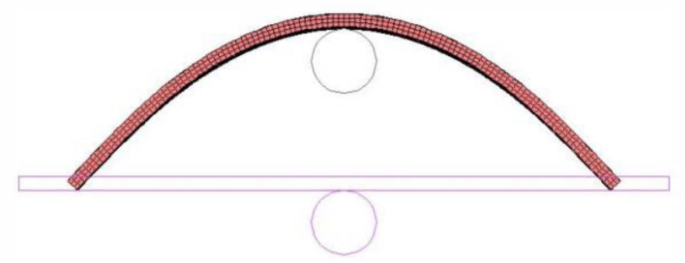

(c)

Figure 10: simulation model unbended (a), cylinder moved upwards 12,5mm (b), cylinder moved $25 \mathrm{~mm}$ upwards (c).

The curvature of the bended interconnection changes along the interconnection, with a maximum curvature at the location where the cylinder touches the interconnection, Figure 10 and Appendix A.

To compare the deformability of interconnections under transversal load the work needed to cause a certain degree of bending, within our investigation the maximum curvature, will be monitored.

\section{B. Results}

Figure 11 shows the work needed to bend the interconnection structure to a certain maximum curvature. This has been done for both interconnection shapes and for 6 different encapsulation thicknesses, these are $0 \mu \mathrm{m}, 250 \mu \mathrm{m}, 500 \mu \mathrm{m}, 750 \mu \mathrm{m}, 1000 \mu \mathrm{m}$ and $2000 \mu \mathrm{m}$.

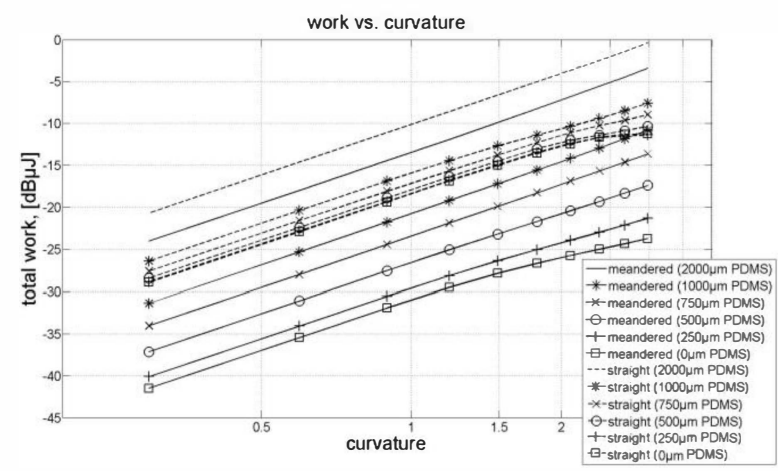

Figure 11: total work needed in function of the maximum curvature

In Appendix $\mathrm{B}$ it shows out that for small deflections the work to bend an interconnection has the following form: 


$$
\begin{gathered}
10 \log _{10} W=10 \log _{10}(E I)+10 \log _{10}\left(\frac{3}{2 l^{3}}\right) \\
+20 \log _{10}(y)
\end{gathered}
$$

Where W, EI, 1 and y indicates respectively the work to bend the interconnection to a certain curvature, EI the flexural rigidity, 1 the half of the interconnection length and $y$ the deflection of the interconnection.

The flexural rigidity, EI, depends on the encapsulation thickness and the shape of the interconnection. A difference in EI can be observed in Figure 11 as an offset between two curves with different encapsulation thickness and/or interconnection shape. The second term is constant for all the different structures that have been tested due to the fact that the length of the interconnections is constant. The third term is the dependency of the deflection; this term will cause an increase of $20 \mathrm{~dB}$ per decade of deflection for small deflections. In Figure 11 an increase of work of about $20 \mathrm{~dB}$ per decade curvature can be observed. This can be justified by the linear relationship between curvature and deflection for small displacements, see Appendix A.

Table 4 compares the work of the two investigated shapes with the same encapsulation thickness. It can be seen that the smaller the encapsulation is, the higher the influence of the interconnection shape becomes. The thicker the encapsulation thicknesses become the less influence the interconnection shapes will have, nevertheless at a rather thick encapsulation thickness of $2 \mathrm{~mm}$, a difference in work of a factor 2 can still be noticed between the different interconnection shapes.

\begin{tabular}{lll}
\hline $\begin{array}{l}\text { Encapsulation } \\
\text { thickness }\end{array}$ & $\begin{array}{l}\Delta \mathrm{dB} \text { (straight } \\
- \text { meandered) }\end{array}$ & $\begin{array}{l}\text { Multiplication } \\
\text { factor }\end{array}$ \\
\hline $0 \mu \mathrm{m}$ & $12,54 \mathrm{~dB}$ & 17,95 \\
\hline $250 \mu \mathrm{m}$ & $11,2 \mathrm{~dB}$ & 13,18 \\
\hline $500 \mu \mathrm{m}$ & $8,68 \mathrm{~dB}$ & 7,38 \\
\hline $750 \mu \mathrm{m}$ & $6,44 \mathrm{~dB}$ & 4,41 \\
\hline $1000 \mu \mathrm{m}$ & $4,97 \mathrm{~dB}$ & 3,14 \\
\hline $2000 \mu \mathrm{m}$ & $3,38 \mathrm{~dB}$ & 2,18 \\
\hline
\end{tabular}

Table 4: comparison of total work needed to bend encapsulated metal interconnects with identical encapsulation but different shape

The obtained results and equations from Appendix B make it possible to determine the flexural rigidity, EI, based on the numerical simulation and following equation:

$$
E I=\frac{2 W l^{3}}{3 y^{2}}
$$

The results for the flexural rigidity based on numerical simulation are depicted in Table 5. Remark that the calculated EI is based on the work and deflection at the first iteration, $y=2,5 \mathrm{~mm}$; because for this iteration the introduced errors due to simplified beam theory are negligible. It can be observed that for meander-shaped interconnections a significant difference in flexural rigidity is observed if the encapsulation thickness increases. For straight tracks this is not the case. The reason behind this behavior is that the initial stiffness of a straight interconnection without encapsulation is that high that the amount of encapsulation already needs to become very high if it needs to become predominant. Where for the stretchable interconnection a significant lower initial stiffness, of a factor 17 , has been observed in comparison with the straight legs. This brings along that the stretchable interconnection its flexural rigidity is mostly determined by the encapsulation and only limited the shape.

\begin{tabular}{lll}
\hline $\begin{array}{l}\text { Encapsulation } \\
\text { thickness }\end{array}$ & Shape & $\begin{array}{l}\text { Flexural rigidity } \\
\mu \mathrm{Jm}\end{array}$ \\
\hline $0 \mu \mathrm{m}$ & Meander & 6 \\
\hline $250 \mu \mathrm{m}$ & Meander & 8 \\
\hline $500 \mu \mathrm{m}$ & Meander & 16 \\
\hline $750 \mu \mathrm{m}$ & Meander & 33 \\
\hline $1000 \mu \mathrm{m}$ & Meander & 62 \\
\hline $2000 \mu \mathrm{m}$ & Meander & 337 \\
\hline $0 \mu \mathrm{m}$ & Straight & 108 \\
\hline $250 \mu \mathrm{m}$ & Straight & 111 \\
\hline $500 \mu \mathrm{m}$ & Straight & 121 \\
\hline $750 \mu \mathrm{m}$ & Straight & 147 \\
\hline $1000 \mu \mathrm{m}$ & Straight & 195 \\
\hline $2000 \mu \mathrm{m}$ & Straight & 734
\end{tabular}

Table 5: flexural rigidity in function of the shape and the encapsulation thickness

\section{Lab experiment}

To validate the simulated results the flexural rigidity of practical test vehicles with different encapsulation thicknesses and interconnection shapes are evaluated by means of a stiffness tester following the normalized test ASTM D1388. This test measures the overhanging length, this is the length at which the end of a test vehicle makes an angle of $41,5^{\circ}$ with the horizontal, Figure 12.

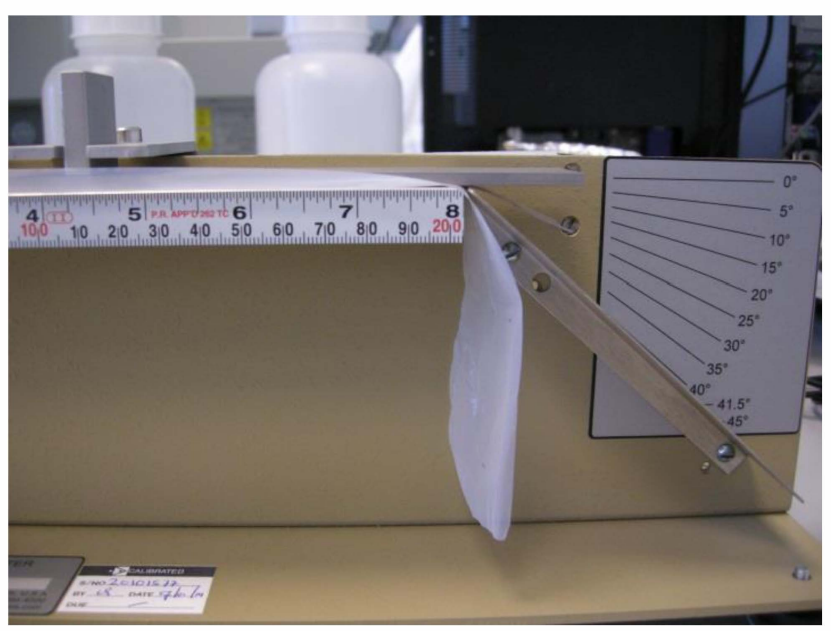

Figure 12: picture of the test environment 
To calculate the flexural rigidity of a test vehicle, the bending length $\mathrm{c}$ needs to be calculated from the overhang length 0 :

$$
c=\frac{o}{2}
$$

The bending length is the half of the overhang length by definition [12].

The flexural rigidity is obtained from the bending length by following formula:

$$
E I=w \cdot c^{3}
$$

Where $\mathrm{w}$ is the weight of the sample per square meter and $\mathrm{c}$ is the bending length. Giving a flexural rigidity in $\mu \mathrm{Jm}$.

The measured overhang lengths, weights and thicknesses of the samples are indicated in Appendix C.

Next table gives the results for the different encapsulation thicknesses and interconnection shapes. The indicated values are calculated out of the averaged results over 3 samples per given structure. Furthermore, the overhang length per sample is measured 4 times, as described in ASTM D1388, and averaged.

A significant correlation between practical results can be observed, despite the minor differences in thickness between the practical and simulated samples.

\begin{tabular}{lll}
\hline $\begin{array}{l}\text { Encapsulation } \\
\text { thickness }\end{array}$ & Shape & $\begin{array}{l}\text { Flexural rigidity } \\
\mu \mathrm{Jm}\end{array}$ \\
\hline $344 \mu \mathrm{m}$ & Meander & 9,7 \\
\hline $456 \mu \mathrm{m}$ & Meander & 17,9 \\
\hline $630 \mu \mathrm{m}$ & Meander & 32,1 \\
\hline $280 \mu \mathrm{m}$ & Straight & 85,7 \\
\hline $470 \mu \mathrm{m}$ & Straight & 124,9 \\
\hline $630 \mu \mathrm{m}$ & Straight & 147,9 \\
\hline
\end{tabular}

Table 6: Flexural rigidity for the different tested test vehicles

Remark: the thickness of the samples has been measured using ASTM D3767 method. By using a normalized weight of $212 \mathrm{~g}$ and a presser foot with a radius of $16 \mathrm{~mm}$.

\section{Conclusions}

Novel insights have been gained regarding the performance of stretchable and flexible metal interconnections to deform under a transversal load. These insights were gained from numerical simulation and verified by practical measurements.

It was proven in the preceding discussion that stretchable shapes have a significant comfort advantage over flexible shapes if they are bent. Up to more then 10 times less work is needed from the environment to bend a stretchable interconnection in comparison with a straight interconnection.
Furthermore an increase of $100 \%$ work per increase in thickness of $250 \mu \mathrm{m}$ was observed for the meandered interconnections. For the straight interconnections the shape of the interconnection is predominant for normal encapsulation thicknesses, $250 \mu \mathrm{m}-1000 \mu \mathrm{m}$, and only a slight increase of max. $20 \%$ per additional $250 \mu \mathrm{m}$ encapsulation is perceived. If the encapsulation thickness increases significantly, $>1 \mathrm{~mm}$, the shape of the interconnection will have less influence on the flexural rigidity of the structure in comparison with smaller encapsulation thicknesses, suggesting to use an encapsulation which is as thin as possible to obtain a comfortable interconnection.

The conclusions regarding encapsulation thicknesses and interconnections shapes are valid but not limited to this case of interconnections. Other elastomers, conductors, support layers can be considered as well as other processing techniques.

From these observations the claim that stretchable interconnections are in general more applicable for wearable, biomedical and textile applications is justified.

\section{Acknowledgments}

The authors would like to thank Sheila Dunphy and Steven Van Put for their practical support in the production of the test vehicles, Dow Corning for supplying Sylgard 186 and the European Commission for the financial support through the PASTA project (Grant Agreement 258724)

\section{Appendix A: simplified beam theory}

The reasoning about the behavior of the interconnection under bending can be better understood when an equivalent beam model is used. If one cuts the model of Figure $10(\mathrm{c})$ in the middle the equivalent schematic of Figure 13(a) is obtained. This schematic can be converted on its terms to one other schematic, Figure 13(b). If one now neglects the displacement in de $\mathrm{x}$-direction, $\Delta \mathrm{x}$, a model is obtained which is easier to analyze and widely discussed in the basic beam theory [11]. Remark that $\Delta \mathrm{x}$ is only small for rather small deflections, in this investigation this will be the case for the first 3-4 iterations as can be seen from the linearity in Figure 11.

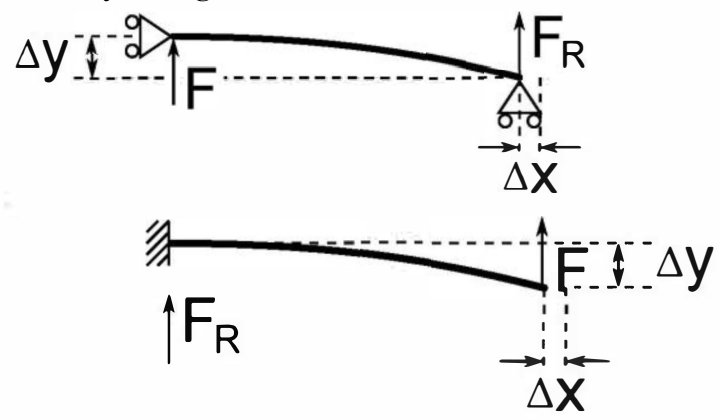

Figure 13: equivalent schematic of a half encapsulated interconnection 
The curvature of a beam subjected to bending is given by:

$$
\kappa=\frac{1}{R}=\frac{M}{E I}
$$

The moment induced at a certain location $\mathrm{x}$ along the interconnection, see the equivalent schematic in Figure 13, is defined as:

$$
M=F(x-l)
$$

After introducing the last equation in the equation of the curvature, we obtain:

$$
\kappa=\frac{1}{R}=\frac{F(x-l)}{E I}
$$

With a maximum curvature at the location $\mathrm{x}=0$ :

$$
\kappa_{\max }=\frac{1}{R_{\min }}=\frac{F l}{E I}
$$

Within the simulation model the location of $x=0$ in the equivalent model is the location where the cylinder touches the interconnection. From this point it is justified that the maximum curvature occurs at this location.

The applied force within the simulation environment is dependent on the deflection of the beam, $y_{\max }$. The maximum deflection of a cantilever is given by:

$$
y_{\max }=\frac{F l^{3}}{3 E I}
$$

Introducing the equation for the deflection in the last equation, we obtain:

$$
\kappa_{\max }=\frac{1}{R_{\min }}=\frac{3 y_{\max }}{l^{2}}
$$

\section{Appendix B: determination of flexural rigidity}

Work is defined in general by the following formula:

$$
W=\int_{C} \vec{F} d \vec{r}
$$

Where $\vec{F}$ is the force vector, in this case the force is only in the y-direction, and $\vec{r}$ is the displacement vector. The nodes where forces are applied are at the end of the interconnection, Figure 13(b). Furthermore, the displacement of these nodes of the interconnection moves in the $\mathrm{x}$ - as well as in the y-direction. To simplify previous equation the dot product of $\vec{F} \cdot d \vec{r}$ and due to the knowledge that there is only a force in the y-direction at the end of the interconnection, the equation for the dissipated work becomes:

$$
W=\int_{0}^{y} F_{l}(y) \cdot d y
$$

Where $\mathrm{W}$ is the total work, $\mathrm{F}_{1}$ is the force applied by the load at the end of the cantilever. It is generally known from the beam theory that the displacement of a beam is dependent on the applied force or vise versa:

$$
\begin{gathered}
W=\int_{0}^{y} \frac{3 E I}{l^{3}} y \cdot d y \\
W=\frac{3 E I y^{2}}{2 l^{3}}
\end{gathered}
$$

If the logarithm is taken of this equation we become:

$$
\begin{gathered}
10 \log _{10} W=10 \log _{10}\left(\frac{3 E I}{2 l^{3}} \cdot y^{2}\right) \\
10 \log _{10} W=10 \log _{10}(E I)+10 \log _{10}\left(\frac{3}{2 l^{3}}\right) \\
+20 \log _{10}(y)
\end{gathered}
$$

Appendix C: measurement results ASTM D1388

\begin{tabular}{llll}
\hline $\begin{array}{l}\text { Encapsulation } \\
\text { thickness }\end{array}$ & Shape & $\begin{array}{l}\text { Weight } \\
{\left[\mathrm{g} / \mathrm{m}^{2}\right]}\end{array}$ & $\begin{array}{l}\text { Overhanging } \\
\text { length }[\mathrm{mm}]\end{array}$ \\
\hline $344 \mu \mathrm{m}$ & Meander & 450 & 26 \\
\hline $456 \mu \mathrm{m}$ & Meander & 600 & 29 \\
\hline $630 \mu \mathrm{m}$ & Meander & 800 & 32 \\
\hline $280 \mu \mathrm{m}$ & Straight & 420 & 55 \\
\hline $470 \mu \mathrm{m}$ & Straight & 550 & 57 \\
\hline $630 \mu \mathrm{m}$ & Straight & 725 & 55 \\
\hline
\end{tabular}

Table 7: Flexural rigidity for the different tested test vehicles

\section{References}

[1] T. Someya, Stretchable Electronics, 2012, Wiley, pp.1-484

[2] B. Balakrisnan, "Design of compliant meanders for applications in MEMS, actuators, and flexible electronics", Smart Materials and Structures, vol. 21, issue 7, Jul. 2012.

[3] C. Pang, "Recent advances in flexible sensors for wearable and implantable devices," Journal of Applied Polymer Science, Vol. 130, Issue 3, pp. 1429-1441, Nov. 2013.

[4] Yung-Yu Hsu, "Novel Strain Relief Design for Multilayer Thin Film Stretchable Interconnects," IEEE Transactions on Electron Devices, Vol. 60, Issue 7, Jul. 2013.

[5] L. Ming, "Design of two-dimensional horseshoe layout for stretchable electronics systems," Journal of Material Science, Vol. 48, Issue 24, Dec. 2013.

[6] A. Jahanshahi, "Stretchable circuits with horseshoe shaped conductors embedded in elastic polymers," Japanese Journal of Applied Physics, Vol. 52, Issue 5, May 2013.

[7] F. Bossuyt, "Stretchable electronics technology for large area applications: fabrication and mechanical characterization," IEEE Transactions on Components, Packaging and Manufacturing 
Technology, Vol. 3, Issue 2, pp. 229-235, Feb. 2013.

[8] J. Vanfleteren, "Printed circuit board technology inspired stretchable circuits," MRS Bulletin, Vol. 37, Issue 3, pp. 254-260, Mar. 2012

[9] Yung-Yu Hsu, "Polyimide-Enhanced Stretchable Interconnects: Design, Fabrication and Characterization", IEEE Transactions on Electron Devices, Vol. 58, No. 8, Aug. 2011.

[10] Yung-Yu Hsu, "The effect of encapsulation on deformation behavior and failure mechanisms of stretchable interconnects," Thin Solid Films, 519(7), p. 2225-2234.

[11] J. Shigley, "Mechanical Engineering Design", International Edition, McGraw Hill, 1986, ISBN 0-07-100292-8

[12] P. Szablewski, "Numerical Analysis of Peirce's Cantilever Test for the Bending Rigidity of Textiles," Fibres and Textiles in Eastern Europe, Oct./Dec. 2003. 